\title{
Thank you to our reviewers
}

The BDJ is always looking for new reviewers to assess manuscripts in all areas of dentistry.

If you would like to be added to our reviewer database, please contact any of the editorial team:

Rowena Milan: r.milan@nature.com

Kate Maynard:k.maynard@nature.com

Stephen Hancocks: bdj@bda.org

The $B D J$ editorial team would like to thank all the people who help maintain the quality of scientific papers in the $B D J$ by acting as reviewers. The following list contains the referees who have worked on scientific papers during 2010.

Liam Addy

Aws Alani

Sondos Albadri

Simon Allum

George Aristidou

Peter Ash

Paul Ashley

Paul Averley

Jeremy Bagg

Graham Ball

Mary Colette Balmer

Bill Barrett

David Bartlett

Paul Batchelor

Garmon Bell

Malcolm Bishop

Igor Blum

Stephen Bonsor

Peter Brennan

Paul Brocklehurst

William Browning

Lucy Burbridge

Trevor Burke

Lyndon Cabot

Peter Carrotte

Luke Cascarini

Robert Chate

Ivor Chestnutt

Robert Clark

Valerie Clerehugh

Joanne Collins

John Cottingham

StJohn Crean

David Croser

Julia Csikar

Chris Cunningham

Gillian Davies

Christopher Dean

Derek Debuse

Debra Dixon

Serpil Djemal

Gail Douglas

John Drummond

Stephen Dunne

Justin Durham

Thomas Dyer

Helen Falcon
Vickie Firmstone

Peter Galgut

Jennifer Gallagher

Dien Gambon

Matthew Garnett

Amargit Gill

Jennifer Godson

Nicholas Goodger

Siobhan Grant

Mark Greenwood

Tony Griffin

Dan Haas

Rebecca Harris

Ken Hemmings

Charles Hildebolt

Martin Hobdell

Nic Hodson

Richard Holmes

Li Wen Hu

Lindsey Hunter

Richard lbbetson

Robert Ireland

Laurence Jacobs

Rob Jagger

Waseem Jerjes

Chris Johnston

Asbjorn Jokstad

Derek Jones

Judith Jones

Rajendra Joshi

Vinod Joshi

Andrzej Juszczyk

Richard Kahan

Anup Karki

Elizabeth Kay

Daniel Knibb

Nigel Knott

Kerstin Knutsson

Jason Leitch

Liran Levin

Ronnie Levine OBE

Debbie Lewis

Graeme Lillywhite

Martin Ling

Yaling Liu

L. P. Longman

Victor Lopes
Victoria Lucas

Christopher Lynch

Mervyn Lyons

Avril Macpherson

Jane Macpherson

Stanley Malamed

George Markose

Michael Martin

Roger Matthews

James McCaul

Fraser McCord

Shane McCrea

Christine McCreary

Robert McGeoch

Mark McGurk

Niall McLeod

Alex McMahon

John Meechan

Christopher Mercer

Brian Millar

Douglas Miller

John Milne

Alex Milosevic

Paul Moore

Simon Moore

Christopher Morris

Karwan Moutasim

James Murphy

Daniel Myers

Tim Newton

Richeal Ní Ríordáin

Paul Nixon

Richard G. Oliver

Richard J. Oliver

Niek Opdam

Christopher Orr

Richard Palmer

S. Parekh

Benjamin Peretz

Nicolaos Pigadas

M. Anthony Pogrel

Neil Poyser

Alison Qualtrough

David Radford

Sobia Rafique

Christoph Ramseier

Michael Reeson
Sue Reeves

John Renshaw

Patricia Reynolds

Scott Rice

Wayne Richards

Graham Roberts

Peter Robinson

Margaret Ross

Vivian Rushton

Stefanie Russell

Jonathan Sandy

Suzanne Scott

Amalia Scuderi

Robin Seymour

Aubrey Sheiham

Jonathan Shepherd CBE

Gill Smith

Michael Smith

Liviu Steier

Tom Thayer

Phillip Tomson

Mônica Tostes

Christopher Tredwin

Alexandre Vieira

Ellen Vinge

Damien Walmsley

Johnny Wang

Mike Wanless

Pamela Ward

Saman Warnakulasuriya

Keith Webster

Richard Welbury

Kathy Wilson

Nairn Wilson CBE

Alain Woda

Geoffrey Wood

Graeme Wright

Xiao Xiangjun

Callum Youngson

Yehuda Zadik

Halla Zaitoun

Thank you also to anyone who reviewed a manuscript towards the end of this year whose name may not appear above. 